\title{
Genèse et évolution du marqueur discursif de toute façon et de ses variantes
}

\author{
Simon Tuchais ${ }^{1 *}$ \\ ${ }^{1}$ Université Sophia, 7-1 Kioi-chô, Chiyoda-ku, Tokyo 102-8554 Japon
}

\begin{abstract}
Résumé. Cette étude se propose de mettre en lumière la genèse du marqueur discursif de toute façon par un examen de ses occurrences dans le corpus Frantext. Elle s'appuie notamment sur les travaux de Combettes (1995), et les poursuit sur une période plus étendue et par une approche quantitative, portant également sur les diverses variantes du marqueur. L'examen montre que c'est dans l'entre-deux-guerres que ce fonctionnement du syntagme prépositionnel s'est imposé, à l'issue d'une série de changements que l'on peut voir comme un processus de constructionnalisation (Traugott \& Trousdale, 2013).
\end{abstract}

\begin{abstract}
The genesis and evolution of discourse marker de toute façon and its variants. This study sheds light on the genesis of French discourse marker de toute façon by examining its occurrences in the Frantext corpus. The study builds on the results of Combettes (1995) by considering a larger period and using a quantitative approach that covers the various variants of the marker. The examination shows that the discourse marker use of de toute façon became prevalent during the interwar period after a series of changes; this process can be seen as a case of constructionalization (Traugott \& Trousdale, 2013).
\end{abstract}

\section{Introduction}

Le syntagme prépositionnel de toute façon présente en français contemporain un fonctionnement de marqueur discursif, ou connecteur argumentatif. Cette étude a pour but de montrer ce que l'examen du corpus Frantext permet de comprendre de la genèse de ce fonctionnement, ainsi que du rapport de de toute façon avec ses variantes.

Le fonctionnement de de toute façon comme connecteur en français contemporain a fait l'objet de diverses descriptions. Rossari $(2000: 85)$ y voit un connecteur de la catégorie des « reformulatifs non paraphrastiques» (Roulet, 1987), dont le propre est «d'indiquer que l'entité Y a pour fonction de rétrointerpréter l'entité X». Dans le cas de de toute façon, il s'agit d'une « rétrointerprétation remettant en cause la valeur rhétorique de X (orientation argumentative, force illocutoire, effet perlocutoire)» (ibid.). Dans un cadre argumentatif, Alvarez-Prendes (2013) propose comme glose : «l'énonciation de X n'est pas retenue comme argument pour $\mathrm{Y} »$. Grieve (1996: 218) lui attribue comme caractéristique principale que « the point introduced by it is always presented as a fact or a certainty ».

\footnotetext{
*s-tuchai@sophia.ac.jp
} 
Le fonctionnement ainsi décrit n'est pas prévisible à partir du sens des éléments qui constituent ce marqueur. La question de son origine a déjà été étudiée par Combettes (1995), qui montre qu'il s'agit du résultat d'un processus de grammaticalisation. Nous poursuivrons ici cette étude sur une durée plus longue, par une approche quantitative, en ayant notamment recours au cadre proposé par Traugott \& Trousdale (2013) pour repenser la grammaticalisation comme un phénomène de constructionnalisation.

Comme le signale entre autres Grieve (1996), de toute façon a dans cet emploi plusieurs variantes, la plus fréquente étant de toute manière. On trouve également des formes de pluriel, de toutes façons, de toutes manières, ainsi que des formes avec article, de toutes les façons, de toutes les manières. Nous utiliserons ci-dessous l'abréviation DTF pour parler de l'ensemble des six formes, les abréviations $d t f$ et $d t m$ pour de toute $(s)$ façon $(s)$ et de toute $(s)$ manière(s), dtlf et $d t l m$ pour les formes avec article.

\section{Genèse de l'emploi discursif}

\subsection{Le processus de grammaticalisation}

Un aspect du processus de grammaticalisation qui a abouti à la genèse du marqueur discursif de toute(s) façon(s) a déjà été mis en lumière par Combettes (1995). L'auteur aborde de toute façon dans le cadre d'une théorie du changement élaborée par E. C. Traugott dans divers travaux, selon laquelle le changement se produit de la composante propositionnelle du système linguistique vers la composante textuelle, puis vers la composante expressive ou interpersonnelle. S'appuyant sur des textes du XIX ${ }^{\mathrm{e}}$ et du début du $\mathrm{XX}^{\mathrm{e}}$ siècle, il montre que l'on peut observer ces trois étapes dans l'évolution de de toute façon. Celui-ci présente d'abord « un fonctionnement strictement circonstanciel (agir de toutes façons $=$ de toutes les manières possibles) $»($ Combettes, 1995 : 35). Dans l'étape suivante, de toute façon n'est plus rattaché à un constituant particulier, ce qui lui permet de figurer en début d'énoncé, et fait référence au contexte linguistique au sens de « quelles que soient les façons citées » (ibid.), ou « de toutes les manières qui viennent d'être évoquées » (ibid. : 41), comme en (1).

(1) Je ne puis cependant la faire avorter pour la sauver [...] Cependant, quand elle saura, elle se tuera certainement, et l'enfant n'étant pas à terme mourra de sa mort. Donc, de toute façon, il est condamné, ce fruit du crime! (J. Péladan, Le vice suprême, 1884, cité par Combettes, 1995 : 42)

Vient enfin l'étape «pragmatique », illustrée par (2), où la locution relève du domaine énonciatif: « en l'absence d'un contenu qui proposerait clairement plusieurs "façons", la portée de la locution se déplace, pourrait-on dire, sur les actes de parole sous-jacents aux énoncés antérieurs $\gg$ (ibid. : 41).

(2) Ses (= de notre âme) communications avec le réel sont fermées ; aussi ne pouvonsnous jouir de la découverte, il est trop tard. Du reste, de toutes façons, pour que j'en pusse jouir spirituellement, celle-là me faisait trop de peine. (M. Proust, La Fugitive, 1922, cité par Combettes, 1995 : 42)

En nous appuyant sur ces conclusions, nous tenterons de montrer qu'il s'agit de la conjonction de plusieurs évolutions que nous détaillerons en 2.3. Nous reprendrons pour cela l'étude sur une plus longue durée et en incluant les différentes variantes de de toute façon. Nous traiterons cette évolution comme un processus de constructionnalisation (plus précisément de grammaticalisation constructionnelle) au sens de Traugott \& Trousdale (2013), qui repensent le changement linguistique dans le cadre des "grammaires de construction ». La constructionnalisation est définie par les auteurs comme le nouvel appariement entre une forme et un $\operatorname{sens}^{1}$ ("The creation of a form new -meaning new pairing", 
2013 : 1). Cette théorie se caractérise notamment par une approche des changements linguistiques basée sur l'usage (« usage-based») qui accorde donc de l'importance à la fréquence. Une autre spécificité de cette approche est que le langage y est vu comme un réseau. Les constructions peuvent donc être étudiées dans le cadre du réseau qu'elles forment avec les constructions proches.

\subsection{Objet de l'analyse}

L'analyse portera sur les occurrences des six formes correspondant aux différentes combinaisons de de toute(s) (les) façon (s)/manière(s) dans le corpus Frantext. Les résultats de la recherche des différentes séquences ont été triés pour ne garder que les cas où elles ont un fonctionnement autonome adverbial (non argumental). Ont ainsi été écartés les cas où le nom façon/manière est lui-même suivi d'une extension, et les cas où la préposition de relève de la valence d'un verbe. Ont aussi été exclus les emplois comme complément du nom, qui constituent la majorité des occurrences de ces séquences dans les premiers siècles représentés dans le corpus : les noms façon et manière se combinent avec tout pour former un déterminant toutes façons/manières de $\mathrm{N}$, au sens de «toutes sortes de $\mathrm{N}$ », et les séquences de toute(s) (les) façon(s)/manière(s) apparaissent comme complément de détermination dans des structures comme des $\mathrm{N}$ de toutes façons, ou après le verbe avec le pronom en : il y en a de toutes façons. Cet emploi diminue peu à peu et disparait à la fin du $\mathrm{XIX}^{\mathrm{e}}$ siècle, à l'exception de quelques cas résiduels au $\mathrm{XX}^{\mathrm{e}}$ siècle.

Les premières occurrences des emplois adverbiaux apparaissent dès le moyen français, mais leur nombre ne devient significatif qu'aux $\mathrm{XVII}^{\mathrm{e}}$ et $\mathrm{XVIII}^{\mathrm{e}}$ siècles, avant que leur fréquence n'augmente fortement au $\mathrm{XX}^{\mathrm{e}}$ siècle. Le tableau 1 montre la fréquence absolue et la fréquence relative (nombre d'occurrences pour un million de mots) de l'ensemble des formes par siècle.

Tableau 1. Fréquence absolue et fréquence relative (par million de mots) de DTF par siècle.

\begin{tabular}{|l|l|l|l|l|l|l|l|l|l|}
\hline Siècle & $\mathrm{XIII}^{\mathrm{e}}$ & $\mathrm{XIV}^{\mathrm{e}}$ & $\mathrm{XV}^{\mathrm{e}}$ & $\mathrm{XVI}^{\mathrm{e}}$ & $\mathrm{XVII}^{\mathrm{e}}$ & $\mathrm{XVIII}^{\mathrm{e}}$ & $\mathrm{XIX}^{\mathrm{e}}$ & $\mathrm{XX}^{\mathrm{e}}$ & $\mathrm{XXI}^{\mathrm{e}}$ \\
\hline Fréquence absolue & 1 & 5 & 2 & 6 & 51 & 112 & 377 & 4188 & 893 \\
\hline Fréquence relative & 0.7 & 1.2 & 0.5 & 0.8 & 2.4 & 3.6 & 5.9 & 39.0 & 66.1 \\
\hline
\end{tabular}

Si l'on examine de plus près l'évolution en se concentrant sur les $\mathrm{XIX}^{\mathrm{e}}$ et $\mathrm{XX}^{\mathrm{e}}$ siècles, on constate que c'est dans l'entre-deux-guerres que la fréquence d'emploi de DTF a commencé à augmenter rapidement, comme on peut le voir dans la figure 1, qui montre le nombre d'occurrences et la fréquence par décennie.

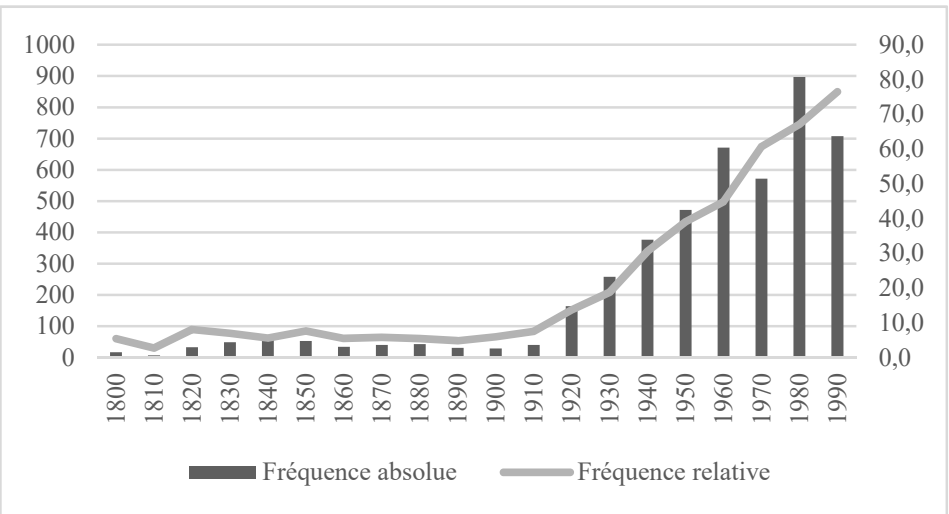

Fig. 1. Fréquence absolue et fréquence relative (par million de mots) de DTF aux XIX et XX ${ }^{\mathrm{e}}$ siècles 
par décennie.

Comme nous le verrons, cette augmentation est due au développement de l'emploi comme marqueur discursif. Afin d'étudier la genèse de cet emploi, nous avons donc effectué un examen détaillé des 1045 occurrences de l'adverbial DTF, toutes formes comprises, jusqu'à 1939.

\section{3 Évolutions observées}

\subsubsection{Du concret vers l'abstrait}

Les noms manière et façon sont utilisés dès l'ancien français avec diverses déterminations pour former des tours adverbiaux introduits par la préposition de servant à indiquer la manière d'effectuer le procès, et les premières occurrences de DTF relèvent de cet emploi. On peut en voir un exemple dans cette occurrence isolée au XIII ${ }^{\mathrm{e}}$ siècle :

(3) Ung jour leur avint qu'ilz encontrerent ung chevalier tout armé d'unes armeures noires, et ses chevaulx estoit plus noirs que meure et sa lance noire. Et il estoit grans et grailles par flans et trop bien taillié de toutes façons au semblant qui dehors en apparut. (La suite du Roman de Merlin, 1235)

Il s'agit certes d'un emploi figuré, mais il accompagne un verbe au sens originellement concret. L'emploi comme circonstant indiquant la manière d'effectuer un procès apparaît plus typiquement dans les occurrences des siècles suivants :

(4) Item, icelui preneur sera tenus de labourer et cultiver bien et deuement, chascun an durant le dit terme, de toutes façons et en cuer de saison toutes les terres et vingne dudit manoir appartenans aus diz religieus [...] (Chartes et documents de l'abbaye de Saint-Magloire, 1330)

Ces emplois représentent la quasi-totalité des occurrences jusqu'au $\mathrm{XVI}^{\mathrm{e}}$ siècle, mais on voit peu à peu apparaître des cas où DTF prend un sens de plus en plus abstrait. En (5), il ne s'agit plus de la manière d'effectuer un procès, et DTF y prend plutôt le sens de « à tous égards ». C'est ce sens que l'on retrouve clairement également en (6).

(5) Car de faict le Roy François avec ce qu'il estoit Royal de toute façon, avoit le nez beau et long, [...] (B. des Périers, Nouvelles récréations et joyeux devis, 1558)

(6) Et certes, la moindre petite consolation de la devotion que nous recevons vaut mieux de toute façon que les plus excellentes recreations du monde. (Saint François de Sales, Introduction à la vie dévote, 1619)

La proportion des emplois avec un sens qui n'est pas strictement celui d'un complément circonstanciel de manière augmente au cours des $\mathrm{XVII}^{\mathrm{e}}$ et $\mathrm{XVIII}^{\mathrm{e}}$ siècles, et ils constituent plus de la moitié des cas au XIX ${ }^{\mathrm{e}}$. Ils peuvent souvent se gloser par «à tous égards », «à tout point de vue », « en tout point », « sur tous les plans », comme en (7) et (8).

(7) Le duc de ..., qui n'aurait pu choisir un gendre plus convenable de toutes façons, accepta avec joie la proposition, arrangea tout avec lui, et quinze jours après nos jeunes gens s'épousèrent. (P. de Marivaux, La Vie de Marianne, 1745)

(8) Il avait deux ans de plus que moi ; il était plus expert dans les armes, plus hardi de toutes façons, plus fort. (Stendhal, L'Abbesse de Castro, 1839)

Cette évolution va même jusqu'à des emplois où, même si le lien est encore clair avec le sens originel de «de toutes les façons possibles», il n'est plus vraiment question d'envisager différentes " manières de faire » ou " manière d'être », ni différents " points de vue », et où DTF semble avoir pour rôle de renforcer le sens du prédicat sur lequel il porte. Il apparaît ainsi dans des contextes où l'on serait tenté de nos jours d'utiliser des adverbes comme complètement ou totalement (9) (10) (11). Tout se passe comme si c'était 
uniquement tout qui livrait le sens lexical, façon et manière ne servant qu'à marquer le rôle adverbial.

(9) Enfin, épuisés de toute façon, nous prîmes congé de nos camarades. (J. de Varenne, Mémoires du chevalier de Ravanne, 1740)

(10) J'ai besoin de repos: je suis plus que fatiguée, je suis excédée de toutes les manières. (J. Cazotte, Le Diable amoureux, 1776)

(11) Ainsi finissons-en donc. Mettons Cosima dans la revue et ne croyez pas que j'attendrai et que l'on me fera aller plus longtemps. Ma patience est à bout. Je suis dégoûtée de toutes façons. (G. Sand, Correspondance, 1840)

DTF est aussi utilisé pour renforcer des prédicats exprimant la nécessité (12) ou la volonté (13), avec un sens proche de « absolument» ou « à tout prix ».

(12) Si sa cousine veut, il la dédommagera, en l'épousant, de la perte qu'elle va faire ; autrement il plaidera, de toute façon il faut qu'il plaide. (Mme Riccoboni, Lettres d'Adelaïde de Dammartin, comtesse de Sancerre, à M. le Comte de Nancé, son ami, 1767)

(13) À John H. Ingram. 87 rue de Rome. Mercredi 11 Août 1875. Cher monsieur, de toutes façons, je veux vous voir! (S. Mallarmé, Correspondance, 1879)

L'évolution observée ci-dessus correspond à un processus de désémantisation, le sens lexical des noms façon et manière devenant de plus en plus abstrait, puis s'effaçant peu à peu pour permettre à DTF d'assumer des rôles de plus en plus variés. Cette évolution n'est cependant pas propre à DTF, et s'observe dans toutes sortes de locutions formées avec ces noms. Dans le cas de DTF, on peut par ailleurs faire l'hypothèse que cette évolution a été favorisée par le déclin d'une autre locution proche, en toutes façons.

\subsubsection{Déclin de En toutes façons}

La locution en toute(s) (les) façon(s)/manière(s), qui présente les mêmes variantes que DTF (nous utiliserons ETF pour désigner l'ensemble des formes), s'est utilisée couramment avec le sens de «à tous égards » bien avant DTF, à une époque où celui-ci s'utilisait essentiellement dans son sens originel.

(14) Car il est forz et hardiz et si est ses parages puissanz en toutes manieres. (La mort le roi Artu, 1230)

On peut même trouver dès le début du $\mathrm{XVI}^{\mathrm{e}}$ siècle quelques occurrences où ETF peut s'interpréter avec le sens du marqueur discursif DTF contemporain.

(15) Pour l'Onuphrius sur platine je ne me souffre pas que la relieure ne vaille rien, pourveu que les marges soient espargnées, car en toute façon le veux je faire de rechef relier en marroquin de levant, selon l'assortiment des autres livres entre lesquels je le veux loger. (N. de Peiresc, Lettres, 1627)

Or, si l'on observe l'évolution de la fréquence d'apparition de ETF dans Frantext, son déclin correspond à la période où DTF voit son sens s'étendre et sa fréquence augmenter. Comme le montre la figure 2, c'est au début du XVIII ${ }^{\mathrm{e}}$ siècle que les courbes des fréquences se croisent. 


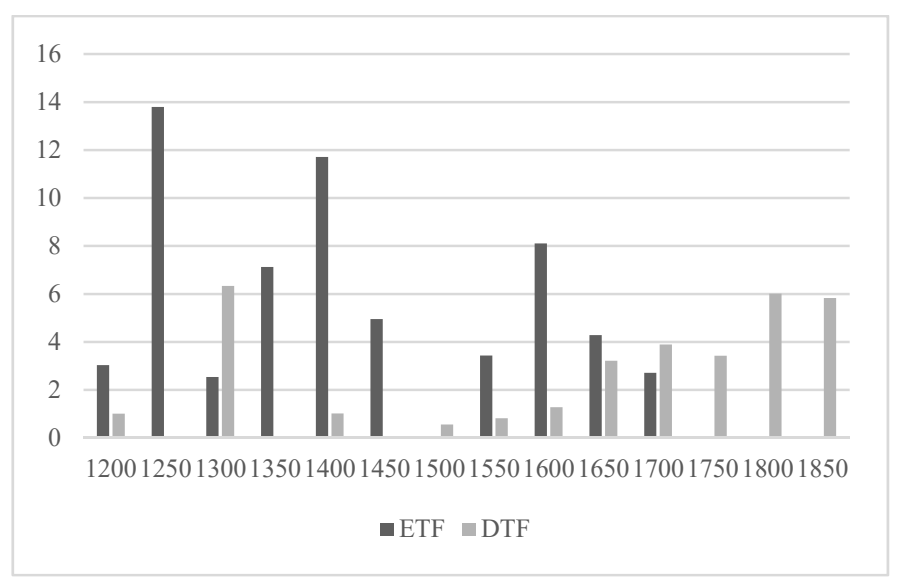

Fig. 2. Fréquence relative (par million de mots) de en toutes façons et de toutes façons et de leurs variantes du XIII ${ }^{\mathrm{e}}$ au $\mathrm{XIX}^{\mathrm{e}}$ siècle par tranches de 50 ans.

Dans les exemples (16) et (17), on peut observer le même verbe complété par en toutes manières au tout début du XVIII ${ }^{\mathrm{e}}$ siècle, puis par de toutes façons, avec un sens similaire, quelques décennies plus tard.

(16) Pour reconnaître donc nos devoirs envers Dieu, comme sagesse ou Raison universelle des intelligences, il ne suffit pas d'être convaincu en toutes manières de l'union de l'esprit avec Dieu, il faut encore examiner avec soin les lois de l'union de l'âme et du corps. (N. Malebranche, Traité de morale, 1707)

(17) Qu'on se figure un homme éperdument amoureux, et jaloux au dernier point, qui a tout à craindre, et qui est convaincu de toutes façons qu'il est échappé au péril qui le menaçoit. (C.-P. Jolyot de Crébillon, L'Écumoire ou Tanzaï et Néadarmé : histoire japonaise, 1734)

$\mathrm{Au}$ vu de ces observations, il est donc probable que le sens de ETF a été pris en charge par DTF, ce qui a contribué à l'extension de ses emplois.

\subsubsection{De l'intraprédicatif à l'extraprédicatif}

Dans son premier emploi, DTF joue un rôle intraprédicatif de circonstant. Il est ainsi souvent coordonné avec d'autres constituants occupant cette fonction, comme dans l'exemple (4) ci-dessus ou comme en (18) :

(18) L'intrigue, ajoutait-il, était aussi nécessaire à Fouché que la nourriture : il intriguait en tout temps, en tous lieux, de toutes manières et avec tous. (E. de Las Cases, Le Mémorial de Sainte-Hélène, 1823)

Mais parallèlement à l'extension de son sens et à son évolution vers l'abstraction, on constate aussi une évolution sur le plan syntaxique dans son rapport avec le prédicat.

Cette évolution s'observe notamment dans le rapport avec la négation. Pour déterminer un prédicat négatif, la forme équivalente attendue est d'aucune façon/manière, ou, dans le sens de «à tous égards », en aucune façon/manière. Ces formes sont effectivement attestées dans des contextes similaires à ceux de DTF, comme on peut le voir en comparant (19) et (20), ou (21) et (22).

(19) Ma pièce est mon bien, je vous le donne ; la postérité même la plus reculée n'en saura rien. Voilà donc votre délicatesse ménagée de toutes façons. (J.-F. Marmontel, Contes moraux, 1761)

(20) C'est-à-dire, que vous ne vous tairez pas sur cette aventure et que vous ne manquerez pas de vous vanter de l'avoir poussée aussi loin qu'il est possible, et de 
ne m’avoir ménagée en aucune façon. (C.-P. Jolyot de Crébillon, La Nuit et le moment ou les Matinées de Cythère, 1755)

(21) Hélas ! mon père, n'avez-vous pas essayé de toutes manières de vous procurer ces treize cents francs? Cela étant impossible, il a bien fallu nous résigner. (E. Sue, Les Mystères de Paris, 1843)

(22) Enfin, n'essayez d'aucune manière, même par pari, d'arriver à moi. (H. de Balzac, Modeste Mignon, 1845)

Ces tours négatifs se sont maintenus jusqu'à nos jours, en aucune façon n'ayant pas connu l'évolution vers l'emploi discursif de DTF et étant resté dans sa fonction de circonstant. Le parallèle entre la forme affirmative de toute façon et les formes négatives en/d'aucune façon s'observe donc tant que les emplois circonstanciels de DTF perdurent. Cependant, dès la fin du XVII ${ }^{\mathrm{e}}$ siècle, ce lien commence à se distendre : on voit en effet apparaître des emplois de DTF dans des contextes négatifs ${ }^{2}$.

(23) Il est même agréable de n'être point tentée de quitter vos belles terrasses. C'est un bonheur pour les goutteux. Ils ne se reprochent point de vous détourner de vos promenades; ils voient qu'on ne saurait être mieux qu'avec eux de toutes manières. (Mme de Sévigné, Correspondance, t. 3 : 1680-1696, 1696)

(24) J'ai prononcé mon propre jugement en blâmant autrefois cet état d'oraison que je confesse aimer aujourd'hui. À cela je n'ai qu'un mot à vous dire, c'est que je ne l'avois pas éprouvé. Je ne prétends pas même le justifier de toutes manières. (J.-J. Rousseau, La Nouvelle Héloïse, 1761)

(25) Il n'est pas ici question de son plus ou moins de mérite : je conviens, d'ailleurs, avec vous, qu'on ne sauroit de toutes façons être plus aimable. (C.-P. Jolyot de Crébillon, Le Hazard du coin du feu, 1763)

On peut voir également un phénomène lié à ce processus de détachement du prédicat dans l'évolution de la position dans la phrase ou la proposition. On observe en effet dans le corpus une augmentation progressive des occurrences en position initiale avec détachement, qui se déroule en parallèle des évolutions signalées jusqu'ici. On peut le constater dans la figure 3, qui montre l'évolution de la proportion de DTF à l'initiale de phrase ou de proposition avec détachement dans l'ensemble des occurrences.

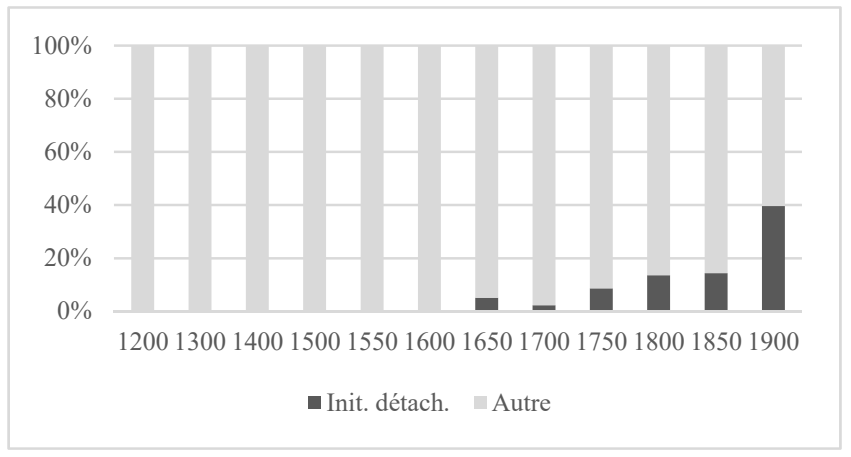

Fig. 3. Proportion des emplois à l'initiale avec détachement par période de 50 ans jusqu'en 1950.

Cette position est certes possible dans sa fonction originelle de circonstant, comme on le constate en (26) ou (27).

(26) Tous les Acteurs, effrayés de cette apparition, renversent la table, les bouteilles, les assiettes, etc. et s'enfuyent en criant. [...] Le Bailli, seul : AH, malheureux ! et ils me brisent tout en s'enfuyant! De toutes les façons, c'est moi qui paie les pots cassés de tout ceci. (C. Collé, La Tête à perruque, ou le Bailli: petit conte dramatique en un petit acte, 1768) 
(27) Avec la renaissance, c'est le cri de sa grandeur et de sa beauté qu'elle fait monter vers le ciel ; avec la réforme, le cri de sa détresse et de sa misère. De toute façon, la créature demande, soit en gémissant, soit en se révoltant, à être réhabilitée. ( $\mathrm{J}$. Maritain, Humanisme intégral : problèmes temporels et spirituels d'une nouvelle chrétienté, 1936)

Mais elle est caractéristique de l'emploi comme marqueur de discours, et favorise l'indépendance de la locution vis-à-vis du prédicat.

\subsubsection{Le rôle du lien avec le contexte dans l'apparition de l'emploi discursif}

Cette possibilité d'apparaître en tête d'énoncé est, comme on l'a vu en 2.1, un phénomène lié à la première étape de la grammaticalisation décrite par Combettes (1995), l'étape « textuelle», dans laquelle DTF joue un rôle dans la cohérence discursive en faisant référence au contexte, illustrée par (1) : cette liberté syntaxique vient de ce que «le groupe prépositionnel ne se trouve plus rattaché à un constituant particulier de la phrase» (Combettes, 1995 : 42). Cependant, un examen sur une période plus étendue et incluant les différentes variantes montre que l'on peut distinguer différents stades dans l'évolution des liens que DTF entretient avec le contexte.

On peut ainsi voir des cas où, dans sa fonction circonstancielle, DTF fait référence à des éléments contextuels, présents dans le même énoncé (28), ou dans l'énoncé suivant (29).

(28) Elle est bien votre fille de toutes façons, non seulement par cette bonne pâte dont vous l'avez faite, mais par le bel et par le bon esprit qu'elle a. (Mme de Sévigné, Correspondance : $t .3: 1680-1696,1696)$

(29) Je prends part de toutes manières à tout ce que vous avez si parfaitement bien fait ; l'amour-propre, l'amitié, la reconnaissance, tout est content. (Mme de Sévigné, Correspondance : t. $3: 1680-1696,1696)$

En (30), l'énoncé suit un paragraphe auquel fait référence un DTF circonstant lié au verbe déchoir, comme le montre la présence de en un mot.

(30) En un mot je déchus de toutes façons [...] (P. de Marivaux, La Vie de Marianne ou les Aventures de madame la comtesse de ***, 1745)

On peut faire la même remarque à propos de $d t l f$ et $d t f$ en (26) et (27) ci-dessus, dont la position initiale est liée au lien avec le contexte même s'ils restent rattachés au verbe. C'est le cas également en (31) :

(31) Travailleuse, cette belle jeunesse voulait le pouvoir et le plaisir ; artiste, elle voulait des trésors ; oisive, elle voulait animer ses passions ; de toute manière elle voulait une place, et la politique ne lui en faisait nulle part. (Honoré de Balzac, Les Illusions perdues, 1843)

On peut voir une évolution par rapport à ces emplois dans les cas où c'est à une alternative que réfère DTF, comme dans l'exemple suivant :

(32) Si je ne dis rien, ils me tueront; si je parle, le capitaine me tuera, ou bien il ne me donnera pas mes vingt mille francs. De toutes façons, je suis volé. Cette alternative peu rassurante étant posée, Rocambole essaya de tourner et de retourner la situation. (P.-A. Ponson du Terrail, Rocambole, les drames de Paris : t. 1, 1859)

Une différence décisive entre ces deux derniers exemples réside dans le fait que, en (31), le procès est effectivement réalisé de toutes les façons évoquées, tandis que, en (32), seul un des termes de l'alternative peut l'être, DTF prenant le sens de «quel que soit le terme de l'alternative choisi ». Il peut prendre dès lors un rôle argumentatif consistant à indiquer que le contenu de l'énoncé qui le contient peut être asserté indépendamment de l'alternative de l'énoncé précédent. Ce type de contexte constitue donc le bridging context (Heine, 2002), qui, tout en permettant l'interprétation dans le sens d'origine, favorise la nouvelle 
interprétation.

Il s'agit en (32) d'une alternative explicite exprimée par le parallèle des deux subordonnées hypothétiques. L'alternative est aussi souvent exprimée par des structures dédiées, comme «que + Subj. ou »:

(33) Que ce soit peur d'être connue, antipathie, orgueil ou malice, de toute manière cette conduite ne mérite qu'une récompense et ne te laisse qu'une attitude à prendre, l'indifférence. (H.-F. Amiel, Journal intime de l'année 1866, 1866)

Sans être aussi clairement présentée, l'alternative peut aussi être reconstituée à partir du contexte, comme c'était le cas en (1). C'est ainsi qu'on peut interpréter également l'un des premiers exemples attestés dans le corpus où une des formes étudiées joue ce rôle :

(34) Tu ne calcules donc aucun des abominables dangers du passage de l'Allemagne. [...] je préfère de beaucoup la traversée de la France. Quoi qu'il en soit, je partirai dès que j'aurai reçu une réponse à la lettre que je t'ai adressée par Alexandre. Elle te priait de me faciliter un voyage plus aisé que celui de l'Allemagne ou de venir audevant de moi jusqu'à Ostende. [...] Encore une fois, de toute manière je partirai, mais j'aimerais cent fois mieux passer par la France s'il en existait un moyen. (Germaine de Staël, Lettres diverses : 1792-1794, 1794)

On voit que $d t m$ y joue déjà un rôle similaire à celui de quoi qu'il en soit, comme le montre la reprise par encore une fois.

Une alternative implicite peut également être introduite par la présence d'une hypothèse, comme celle de la venue du destinataire en (35) :

(35) Si vous êtes libre, venez oublier ici l'ennui des choses matérielles pour ne vous rappeler que le chef-d'œuvre que vous venez de faire et que vous me raconterez en attendant que je le voie. Je suis encore ici pour le reste du mois ; aux premiers jours de novembre, je vais à Paris. De toutes façons, nous nous embrasserons donc bientôt. Mais je serais si heureuse de vous donner ici quelques jours de repos et de distraction! (G. Sand, Correspondance, 1851)

L'évocation d'une possibilité implique une alternative (elle peut être réalisée ou non). DTF peut dans ce cas être glosée par une structure concessive : «même si vous ne venez pas », d'où le rôle argumentatif qu'il assume dès cette étape du processus. On peut penser que c'est ainsi que s'effectue le passage à la troisième étape évoquée par Combettes (1995), où le fonctionnement de DTF se situe au niveau énonciatif (ex. (2)) : l'assertion de $p$ implique la possibilité de non $p$, et c'est à cette alternative que réfère DTF dans cet emploi. Ce passage correspond aussi à celui entre le bridging context et le switch context de Heine (2002) : dans ce dernier, l'interprétation dans le sens d'origine n'est plus possible.

Les cas où DTF suit l'expression d'une alternative explicite représentent une proportion importante - environ $10 \%$ - des occurrences entre 1830 et 1919, c'est-à-dire dans les décennies qui précèdent la période de l'entre-deux-guerres qui verra s'installer DTF dans son emploi contemporain. On peut donc penser que cette fréquence a été un facteur important du processus.

\subsubsection{Diversité des premiers emplois discursifs}

L'évolution décrite dans la section précédente aboutit à l'emploi contemporain du marqueur de discours DTF. Or, on trouve au $\mathrm{XIX}^{\mathrm{e}}$ siècle et au début du $\mathrm{XX}^{\mathrm{e}}$ siècle, époque d'apparition de nouveaux emplois au niveau énonciatif, des occurrences qu'on peut voir comme des innovations ponctuelles.

On trouve ainsi des cas où DTF semble jouer un rôle de modalisateur exprimant la certitude. Cet emploi peut être vu comme découlant du rôle évoqué ci-dessus consistant à souligner la possibilité d'asserter $p$ indépendamment d'une alternative explicite ou non. Ce 
lien est clair dans des exemples comme les suivants, où l'expression de la certitude est explicite :

(36) On ne connaît pas assez complétement les idées des anciens pour dire si ce caractère sacrosaint rendait la personne du tribun honorable aux yeux des patriciens, ou la posait au contraire comme un objet de malédiction et d'horreur. Cette seconde conjecture est plus conforme à la vraisemblance. Ce qui est certain, c'est que, de toute manière, le tribun se trouvait tout à fait inviolable, la main du patricien ne pouvant le toucher sans une impiété grave. (N.-D. Fustel de Coulanges, La Cité antique, 1864)

(37) Pour mes étrennes je suis en grande hésitation. Il y a clairières dans le ciel, l'Agamemnon d'Eschyle, Gide [...] Il y a (combattant Jammes et Gide) Laforgue et Rimbaud. Il y a tant de choses et si peu de temps pour les lire. Ce qui est sûr, c'est que de toutes façons il me faut du Gide. (Alain-Fournier, Correspondance avec Jacques Rivière, 1914)

Or, dans l'exemple suivant, DTF joue seul ce rôle d'expression de la certitude, et ce sans faire référence à une alternative ni s'inscrire dans une argumentation.

(38) - Vous restez encore quelque temps dans la région, madame ? [...]

- Oh! Certainement, monsieur, je tiens beaucoup pour les enfants à cet exode annuel. On a beau dire, il leur faut le grand air. La faculté voulait m'envoyer à Vichy ; mais c'est trop étouffé, et je m'occuperai de mon estomac quand ces grands garçons-là auront encore un peu poussé. Et puis le professeur, avec les examens qu'il fait passer, a toujours un fort coup de collier à donner, et les chaleurs le fatiguent beaucoup. Je trouve qu'on a besoin d'une franche détente quand on a été comme lui toute l'année sur la brèche. De toutes façons nous resterons encore un bon mois.

- Ah! Alors nous sommes gens de revue.

(Marcel Proust, Sodome et Gomorrhe, 1922)

Cet emploi, même s'il est le résultat d'une évolution vers un sens plus pragmatique, ne semble pas avoir perduré jusqu'au français contemporain, ce que l'on peut expliquer, dans le cadre de la constructionnalisation de Traugott \& Trousdale (2013), par le fait que sa faible fréquence n'a pas donné lieu à un processus de conventionnalisation.

Dans ses emplois argumentatifs également, on constate une plus grande diversité dans les rôles assumés. DTF prend place en effet parmi d'autres marqueurs de discours dont le rôle est d'indiquer la possibilité d'asserter un contenu propositionnel indépendamment d'une alternative, comme quoi qu'il en soit ou en tout cas. Or, certains exemples tendent à faire penser que les relations entre ces marqueurs très proches n'étaient pas fixées comme elles le sont de nos jours dans les premiers temps du marqueur DTF ${ }^{3}$. On peut cependant voir dès le $\mathrm{XIX}^{\mathrm{e}}$ siècle et le début $\mathrm{du} \mathrm{XX}^{\mathrm{e}}$ siècle une proportion importante des emplois discursifs de DTF dans des contextes typiques du marqueur contemporain, et on peut faire l'hypothèse que c'est cette association fréquente qui a entraîné sa spécialisation en même temps que son fort développement au milieu du $\mathrm{XX}^{\mathrm{e}}$ siècle. C'est le cas par exemple de contextes dénotant l'inévitabilité (par exemple forcer en (39)), ou comme corollaire, l'inutilité (à quoi bon en (40)).

DTF présente aussi dès cette époque des affinités avec d'autres connecteurs également typiques de son emploi contemporain. L'inévitabilité du procès qu'introduit DTF en fait un argument décisif, et il est ainsi souvent associé à d'ailleurs, qui sert précisément à ajouter un argument (39). Parmi les différents « effets de sens» reconnus à d'ailleurs par Paillard (1991 : 63), il y a en effet celui consistant à « coupe[r] court à toute forme de mise en doute, discussion ou contestation, [...] reproche ».

(39) On veut savoir si nous déménagerons après-demain. Je réponds que je paierai le drôle dans trois jours et que je ne puis mieux faire; que, d'ailleurs, la grippe de nos 
enfants nous forcera, de toutes manières, à différer le déménagement jusqu'à jeudi, peut-être [...] (L. Bloy, Journal 2, 1904)

Comme en français contemporain, il est aussi déjà associé à puisque, qui, parmi les connecteurs de cause, a la particularité de présenter le fait comme déjà admis et hors discussion.

(40) Inutile de demander à Nabucet comment ils étaient. À quoi bon, puisque de toute façon le Capitaine n'y pourrait mettre les pieds. (L. Guilloux, Le Sang noir, 1935)

À l'issue du processus de grammaticalisation, DTF en vient à pouvoir assumer seul ce rôle argumentatif. On peut le voir en comparant (40), où il accompagne puisque pour introduire un argument justifiant l'affirmation de l'inutilité d'un effort, avec (41) et (42), où le marqueur est employé seul dans le même contexte.

(41) Depuis ma chute de bicyclette, une dent me manquait, le trou était visible et je ne songeais pas à le faire combler : à quoi bon? De toute façon, j'étais vieille, j'avais trente-six ans [...]. (S. de Beauvoir, La force des choses, 1963)

(42) À quoi bon, de toute manière avant dix ans, l'un et l'autre seront réduits en poussière. (M. Rheims, Les Greniers de Sienne, 1987)

On peut y voir un cas de ce que Kuteva (2001 : 151) appelle context absorption (voir aussi Traugott \& Trousdale, 2013: 56, 228), phénomène qu'elle décrit ainsi : " the structure absorbs a context with which it frequently co-occurs, so that what before the contextabsorption had to be made explicit in the immediate context no longer needs to be stated: it has been absorbed by the structure itself and has become part of its meaning. »

\section{3 Évolution des différentes formes}

Les différentes combinaisons de de toute(s) (les) façon(s)/manière(s) sont toutes attestées dans les principaux emplois décrits ci-dessus. Ce n'est que dans la chronologie et dans les proportions que l'on observe des différences.

Si l'on considère tout d'abord l'ensemble des emplois de ces syntagmes prépositionnels adverbiaux, même si $d t f$ est le premier attesté dans Frantext en ancien français et domine jusqu'à la première moitié du XVII ${ }^{\mathrm{e}}$ siècle, la seconde moitié voit, en même temps qu'une augmentation du total des occurrences, une diversification, et aucune forme ne s'impose jusqu'au début du $\mathrm{XX}^{\mathrm{e}}$ siècle (figure 4).

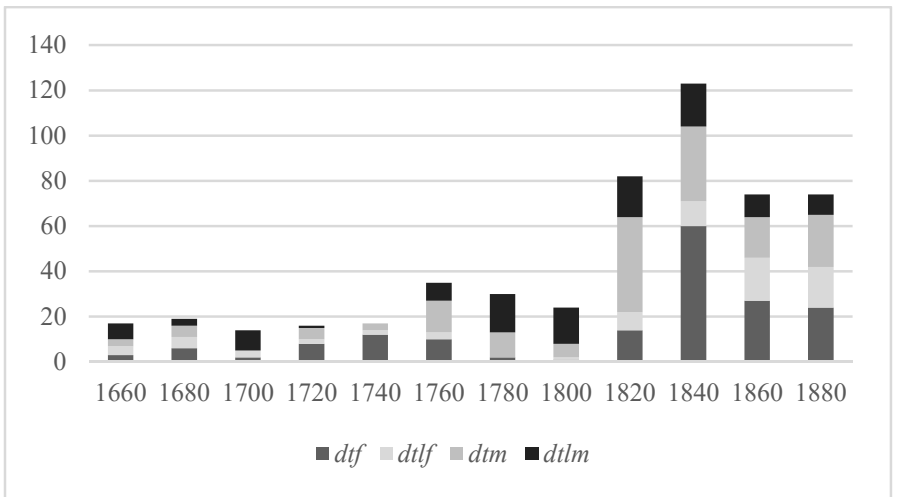

Fig. 4. Fréquence absolue de $d t f, d t l f, d t m$, et $d t l m$ par période de 20 ans de 1660 à 1899 .

C'est à partir du début du $\mathrm{XX}^{\mathrm{e}}$ siècle, et surtout dans l'entre-deux-guerres, que $d t f$ devient très largement majoritaire (figure 5). 


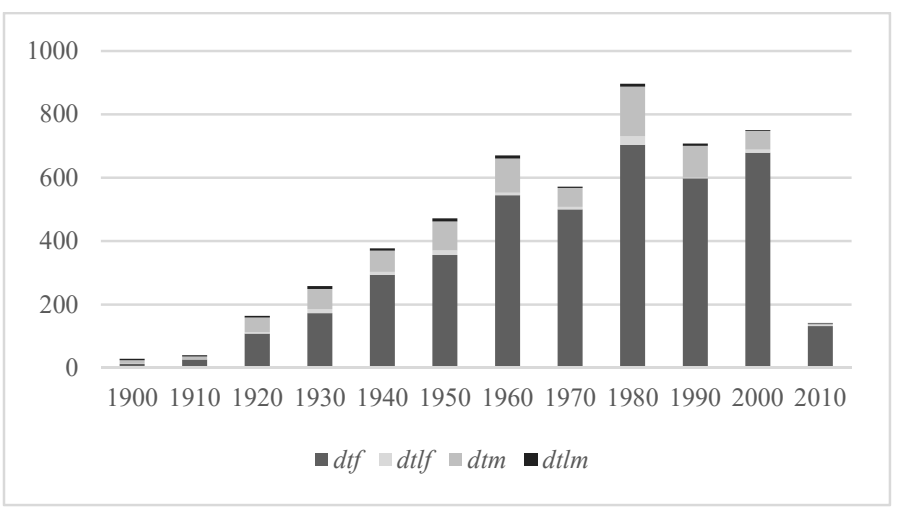

Fig. 5. Fréquence absolue de $d t f, d t l f, d t m$, et $d t l m$ par décennie de 1900 à 2019.

Le nombre d'occurrences de $d t m$ augmente aussi fortement dans les années 1920, mais cesse ensuite de progresser.

Si l'on s'en tient aux emplois discursifs, on observe une hésitation entre $d t f$ et $d t m$ au cours du $\mathrm{XIX}^{\mathrm{e}}$ siècle, mais dès la fin de ce siècle, $d t f$ devient majoritaire dans cet emploi, et c'est sous cette forme que va s'opérer la constructionnalisation dans les années 1920. L'augmentation du nombre d'occurrences durant cette période, observée dans la figure 5, est due à l'augmentation rapide des emplois de $d t f$ comme marqueur discursif. Dans les deux dernières décennies de la période examinée, 1920-1929 et 1930-1939, ces emplois représentent en effet respectivement $81 \%$ et $88 \%$ des occurrences de cette forme (toutes graphies comprises), alors qu'ils ne représentent encore que $48 \%$ et $76 \%$ de celles de $d t m$.

Avec les formes avec articles, la différence est encore plus importante. Les occurrences pouvant s'interpréter comme des emplois discursifs - comme (43) ou (44) - sont beaucoup plus rares que pour $d t f$ et $d t m$ au $\mathrm{XIX}^{\mathrm{e}}$ siècle, particulièrement dans le cas de $d t l m$.

(43) Il lui expliquait à mi-voix que dix mille francs d'un côté et quinze mille francs de l'autre, ça faisait vingt-cinq mille. Une affaire superbe! De toutes les façons, Muffat la lâchait ; c'était un joli tour de force, d'avoir tiré cette dernière plume de son aile. (É. Zola, Nana, 1880)

(44) L'aventure fit du bruit; mes ennemis y virent une indélicatesse, mes amis un trait d'esprit : ce n'était ni l'un ni l'autre ; mais, de toutes les manières, ma position, comme romancier, était détruite. (L. Reybaud, Jérôme Paturot à la recherche d'une position sociale, 1842)

Et sur la période 1920-1939, ils ne représentent que $33 \%$ des occurrences pour dtlf et $14 \%$ pour $d t l m$ - même si le nombre total, respectivement 18 et 14, est trop faible pour que ces proportions soient vraiment significatives. Pour ces deux formes, nous avons poussé l'examen dans le corpus Frantext jusqu'à la période contemporaine, et c'est plus tardivement, à la fin du $\mathrm{XX}^{\mathrm{e}}$ siècle, que les emplois discursifs y deviennent majoritaires. On a donc un ordre $d t f>d t m>d t l f>d t l m$ dans la fréquence et dans la chronologie d'apparition des emplois discursifs.

La théorie de la constructionnalisation de Traugott \& Trousdale (2013) peut fournir un cadre d'interprétation à ces faits. Le réseau de constructions qui constitue le langage a une structure hiérarchique et peut être représenté sous la forme d'une taxinomie. Au plus haut niveau se trouvent les schémas les plus abstraits («macro-constructions » dans les travaux antérieurs de E. C. Traugott \& G. Trousdale), au plus bas les micro-constructions concrètes. Ils donnent pour exemple le cas des quantifieurs en anglais $(2013: 17)$ : au plus haut niveau se trouve le schéma « quantifieur » qui inclut tous les quantifieurs quels qu'ils soient; au niveau intermédiaire se trouvent les sous-schémas «quantifieur de grande quantité » et « quantifieur de petite quantité »; au niveau le plus bas, on trouvera, sous le premier sous- 
schéma many et a lot of, sous le second few et a bit of. Dans le processus de constructionnalisation, la nouvelle construction est «sanctionnée » par un schéma, qui s'étend de ce fait et gagne en schématicité (c'est-à-dire en abstraction) et en productivité. Ainsi, à la suite de la constructionnalisation de a lot of, expression partitive (" un lot de ») qui devient quantifieur («beaucoup de»), « the [quantifier] schema grew as a result of sanctioning the new micro-constructions a lot of, lots of, and appears to have 'motivated' or provided the template for, several other new uses of partitives and measure expressions as quantifiers » (2013 : 49), notamment les « binominaux » (de la forme $a \mathrm{~N}$ of $\mathrm{N}$ ) comme $a$ heap of. En effet, " constructions in the network with similar meaning and form may be important contextual factors and serve as models or attractors » $(2013: 230)$. On peut voir un processus similaire dans le cas de DTF: la nouvelle construction résultant de la conventionnalisation de $d t f$ observée dans les années 1920-1930 est sanctionnée par le schéma "marqueur discursif» et par le sous-schéma "connecteur reformulatif non paraphrastique », et fournit un modèle pour l'évolution de dtm, dtlf, dtlm.

Un autre phénomène tend à corroborer cette approche: l'évolution de n'importe comment. On observe en effet, surtout dans certains registres familiers, une évolution semblable à $d t f$. D'un emploi comme circonstant (issu lui-même d'un premier processus de grammaticalisation le faisant passer d'un statut verbal à un statut adverbial) dénotant lui aussi à la fois la manière et la totalité distributive, il a évolué vers un emploi de connecteur dans lequel il pourrait commuter avec de toute façon :

(45) $[. .$.$] ce qui ne l'empêche pas de se plaindre d'une voix désolée : « Nous parlons$ toujours de moi ! Parlons de vous ! » et bien entendu ça m'ôte toute envie de le faire. N'importe comment je ne l'aurais pas fait, je ne parle quasi jamais de moi à quiconque. (S. de Beauvoir, Lettres à Nelson Algren : un amour transatlantique 1947-1964, 1947)

(46) Les sous-fifres à Lopardi me le répétaient. «Tu ferais mieux de passer à table, les hors-d'œuvre sont servis et le patron te prépare un de ces plats de résistance que tu vas t'en lécher drôlement les babines ! Avec nous, ça sert à rien de faire la fine gueule, le délicat sans appétit! N'importe comment, l'addition c'est toi qui la casqueras. Nous, on est juste comme qui dirait des invités. » (A. Boudard, La Cerise, 1963)

La chronologie suggère que c'est le développement de l'emploi comme marqueur discursif de $d t f$ qui a pu sanctionner cette évolution chez un adverbial dont le sens circonstanciel était proche : si l'on s'appuie sur une analyse des occurrences de n'importe comment dans Frantext, c'est dans les années 1930 que cet emploi commence à apparaître, et il se développe dans la seconde moitié du $\mathrm{XX}^{\mathrm{e}}$ siècle (il représente plus de $10 \%$ des occurrences de l'adverbe sur cette période), suivant ainsi l'évolution de $d t f$.

Si l'on se penche pour finir sur la différence entre les formes de pluriel de toutes façons/manières et de singulier de toute façon/manière, on constate qu'elles sont les unes comme les autres attestées dans tous les emplois successifs, mais que l'évolution des proportions montre des tendances qui peuvent être mises en rapport avec l'évolution des usages. Même s'il ne faut pas se fier outre mesure à la graphie (il arrive qu'on trouve par exemple dans la base Frantext les deux formes pour une même occurrence dans deux éditions différentes d'un même ouvrage), l'expansion des formes de singulier semble en effet suivre chronologiquement l'évolution vers l'abstraction observée en 2.3.1, et surtout le développement de l'emploi comme marqueur discursif, comme on peut l'observer dans la figure 6 . 


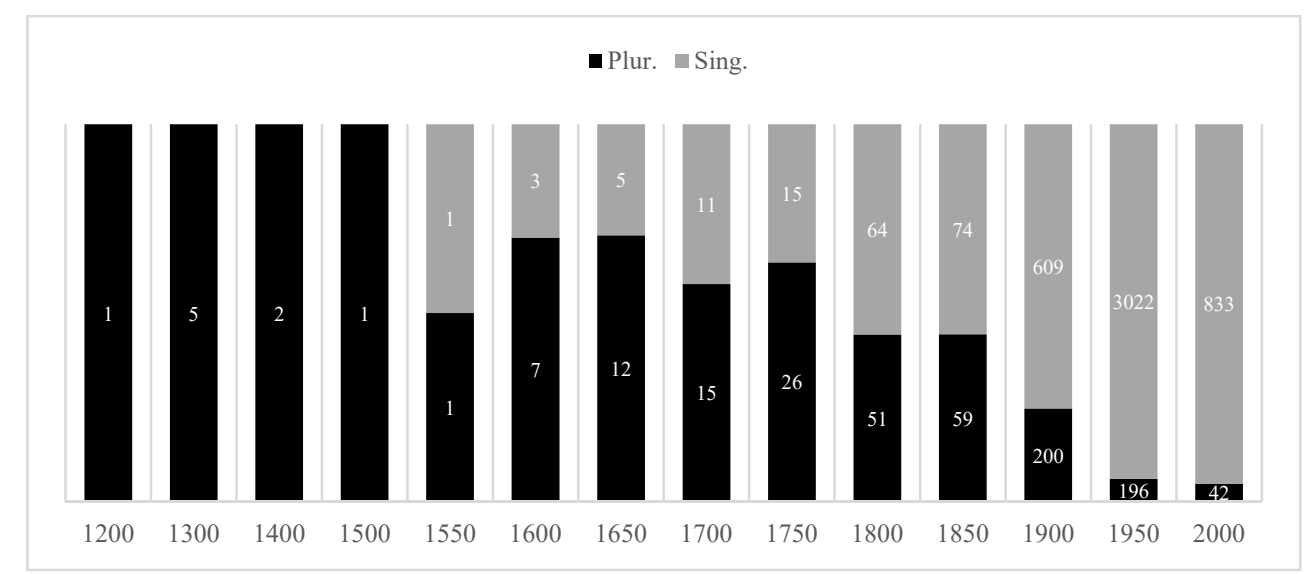

Fig. 6. Proportion des formes de singulier et de pluriel de $d t f$ et $d t m$ par période de 50 ans.

Cette évolution est cohérente avec l'abandon de la référence à une pluralité de «façons » qui accompagne ces changements, ainsi qu'avec le développement du sens argumentatif à partir de la référence à une alternative dont seul un terme peut être réalisé (2.3.4). C'est sans doute aussi de la même manière que l'on peut expliquer la relative marginalisation des formes avec article, qui imposent le pluriel, alors qu'elles ont été majoritaires à une certaine époque.

\section{Conclusion}

L'examen a montré que la genèse du marqueur discursif de toute façon résultait d'une évolution correspondant au processus de constructionnalisation décrit par Traugott \& Trousdale (2013) : une série de « changements constructionnels » (changements affectant seulement une dimension d'une construction) aboutissant à la conventionnalisation d'un nouvel appariement entre une forme et un sens. Nous avons en effet observé une désémantisation graduelle des emplois comme circonstant, principalement à partir du XVI siècle, puis un détachement du prédicat. Ces changements ont rendu possible l'évolution observée par Combettes (1995) au XIX ${ }^{\mathrm{e}}$ siècle et au début du $\mathrm{XX}^{\mathrm{e}}$ siècle : le passage du propositionnel au textuel puis à l'énonciatif. L'examen a permis d'affiner la compréhension de cette évolution, en mettant en lumière l'importance du rôle du contexte : elle s'est en effet produite dans des contextes impliquant une alternative, jouant le rôle de bridging contexts, dans lesquels DTF peut jouer un rôle argumentatif consistant à indiquer que le contenu de l'énoncé qui le contient peut être asserté indépendamment de l'alternative de l'énoncé précédent ; l'étape suivante est celle des switch contexts dans lesquels l'alternative n'est plus explicite et ne permet donc plus la référence à une pluralité de manières, interdisant ainsi l'interprétation dans le sens d'origine. Le processus s'est enfin achevé avec la conventionnalisation du nouvel emploi dans l'entre-deux-guerres.

Cette conventionnalisation s'est opérée d'abord sous la forme de toute façon, qui a fourni un modèle aux autres formes de la catégorie des circonstants dénotant la manière et la totalité ou la totalité distributive : de toute manière, de toutes les façons, etc. Suivant la même évolution celles-ci sont devenues des variantes minoritaires de de toute façon. C'est particulièrement le cas des formes avec article ainsi que des formes de pluriel, ce qui peut s'expliquer par le fait que, référant à la totalité, elles ont moins d'affinités que celles qui réfèrent à la totalité distributive avec le sens discursif qui repose sur l'existence d'une alternative dont seul un terme peut être réalisé. L'étude du rapport entre les différentes variantes devrait cependant être poursuivie pour la période contemporaine d'un point de vue variationniste, en s'appuyant sur l'analyse d'autres corpus de genres variés. 


\section{Références bibliographiques}

Alvarez-Prendes, E. (2013). De toute façon. In J.-C. Anscombre \& P.-P. Haillet, Opérateurs discursifs du français. Éléments de description sémantique et pragmatique. Berne: Peter Lang, 91-96.

Combettes, B. (1995). Approche diachronique des adverbiaux contextuels. Linx, 32, 33-50.

Grieve, J. (1996). Dictionary of contemporary French connectors. New York : Routledge.

Heine, B. (2002). On the role of context in grammaticalization. In I. Wischer \& G. Diewald (éd.), New reflections on grammaticalization. Amsterdam : John Benjamins, 83-101.

Kuteva, T. (2001). Auxiliation: an enquiry into the nature of grammaticalization. New York/Oxford : Oxford University Press.

Paillard, D. (1991). D'ailleurs ou comment enchaîner l'un à l'autre. Le Gré des Langues, 2, 60-65.

Rossari, C. (2000). Connecteurs et relations de discours : des liens entre cognition et signification. Nancy : Presses Universitaires de Nancy.

Roulet, E. (1987). Complétude interactive et connecteurs reformulatifs. Cahiers de linguistique française, $8,111-140$

Traugott, E. C. \& Trousdale, G. (2013). Constructionalization and Constructional Changes. Oxford Oxford University Press.

\footnotetext{
${ }^{1}$ Ces deux dernières notions sont prises dans un sens large: le sens englobe les dimensions sémantiques, discursives, et pragmatiques, tandis que la forme inclut la phonologie et la morphologie mais aussi la syntaxe.

${ }^{2}$ On peut certes considérer que DTF porte sur le second verbe tandis que la négation porte sur le premier, mais en aucune façon/manière est précisément particulièrement fréquent dans ce type de contexte (ne $+\mathrm{V}+\mathrm{INF}+$ en aucune façon/manière), et on en trouve de nombreux exemples à la même époque avec les mêmes verbes :

« Je ne saurais l'adopter en aucune manière. » (E. Sieyès, Euvres, t. 2, 1791)

"Jugeons donc sans balancer, que nos sensations ont en effet hors de nous la cause que nous leur supposons, puisque l'effet qui peut résulter de l'existence réelle de cette cause ne sauroit différer en aucune maniere de celui que nous éprouvons." (D'Alembert, Discours préliminaire de l'Encyclopédie, 1751)

"C'est à la métaphysique et à la théologie qu'il appartient de discuter ces questions, auxquelles nous ne prétendons ici toucher en aucune maniere. » (P. H. Dietrich, baron d'Holbach, La Morale universelle ou les Devoirs de l'homme fondés sur la nature, 1776)

${ }^{3}$ Il serait nécessaire de mener un examen de l'évolution de l'ensemble de ces marqueurs et de leurs rapports en français contemporain, mais celui-ci dépasserait le cadre de la présente étude.
} 